\title{
Extension of Hu Ke's inequality and its applications
}

Jing-Feng Tian

Correspondence:

tianjfhxm_ncepu@yahoo.cn

College of Science and

Technology, North China Electric

Power University, Baoding, Hebe

Province, 071051, People's Republic of China

\section{Abstract}

In this paper, we extend Hu Ke's inequality, which is a sharpness of Hölder's inequality. Moreover, the obtained results are used to improve Hao Z-C inequality and Beckenbach-type inequality that is due to Wang.

Mathematics Subject Classification (2000) Primary 26D15; Secondary 26D10

Keywords: integral inequality, Hölder's inequality, Hu Ke's inequality, Hao Z-C inequality, Beckenbach-type inequality, arithmetic-geometric mean inequality

\section{Introduction}

The classical Hölder's inequality states that if $a_{k} \geq 0, b_{k} \geq 0(k=1,2, \ldots, n), p>0, q>0$ and $\frac{1}{p}+\frac{1}{q}=1$, then

$$
\sum_{k=1}^{n} a_{k} b_{k} \leq\left(\sum_{k=1}^{n} a_{k}^{p}\right)^{\frac{1}{p}}\left(\sum_{k=1}^{n} b_{k}^{q}\right)^{\frac{1}{q}} .
$$

The inequality (1) is reversed for $p<1(p \neq 0)$. (For $p<0$, we assume that $a_{k}, b_{k}>0$.) The following generalization of (1) is given in [1]:

Theorem A. (Generalized Hölder inequality). Let $A_{n j} \geq 0, \sum_{n} A_{n j}^{\lambda_{j}}<\infty, \lambda_{j}>0(j=1$, $2, \ldots, k)$. If $\sum_{j=1}^{k} \frac{1}{\lambda_{j}}=1$, then

$$
\sum_{n} \prod_{j=1}^{k} A_{n j} \leq \prod_{j=1}^{k}\left(\sum_{n} A_{n j}^{\lambda_{j}}\right)^{1 / \lambda_{j}} .
$$

As is well known, Hölder's inequality plays a very important role in different branches of modern mathematics such as linear algebra, classical real and complex analysis, probability and statistics, qualitative theory of differential equations and their applications. A large number of papers dealing with refinements, generalizations and applications of inequalities (1) and (2) and their series analogues in different ares of mathematics have appeared (see e.g. [2-30] and the references therein).

Among various refinements of (1), $\mathrm{Hu}$ in [13] established the following interesting theorems.

\section{Springer}

(c) 2011 Tian; licensee Springer. This is an Open Access article distributed under the terms of the Creative Commons Attribution License (http://creativecommons.org/licenses/by/2.0), which permits unrestricted use, distribution, and reproduction in any medium, provided the original work is properly cited. 
Theorem B. Let $p \geq q>0, \frac{1}{p}+\frac{1}{q}=1$, let $a_{n}, b_{n} \geq 0, \sum_{n} a_{n}^{p}<\infty, \sum_{n} b_{n}^{q}<\infty$, and let $1-e_{n}+e_{m} \geq 0, \Sigma_{n}\left|e_{n}\right|<\infty$. Then

$$
\begin{aligned}
\sum_{n} a_{n} b_{n} \leq\left(\sum_{n} b_{n}^{q}\right)^{\frac{1}{q}-\frac{1}{p}}\left\{\left[\left(\sum_{n} b_{n}^{q}\right)\left(\sum_{n} a_{n}^{p}\right)\right]^{2}\right. \\
\left.\quad-\left[\left(\sum_{n} b_{n}^{q} e_{n}\right)\left(\sum_{n} a_{n}^{p}\right)-\left(\sum_{n} b_{n}^{q}\right)\left(\sum_{n} a_{n}^{p} e_{n}\right)\right]^{2}\right\}^{\frac{1}{2 p}} .
\end{aligned}
$$

The integral form is as follows:

Theorem C. Let E be a measurable set, let $f(x)$ and $g(x)$ be nonnegative measurable functions with $\int_{E} f^{p}(x) \mathrm{d} x<\infty, \int_{E} g^{q}(x) \mathrm{d} x<\infty$, and let $e(x)$ be a measurable function with $1-e(x)+e(y) \geq 0$. If $p \geq q>0, \frac{1}{p}+\frac{1}{q}=1$, then

$$
\begin{gathered}
\int_{E} f(x) g(x) d x \leq\left(\int_{E} g^{q}(x) d x\right)^{\frac{1}{q}-\frac{1}{p}}\left[\left(\int_{E} f^{p}(x) d x \int_{E} g^{q}(x) d x\right)^{2}-\right. \\
\left.\left(\int_{E} f^{p}(x) e(x) d x \int_{E} g^{q}(x) d x-\int_{E} f^{p}(x) d x \int_{E} g^{q}(x) e(x) d x\right)^{2}\right]^{\frac{1}{2 p}} .
\end{gathered}
$$

The purpose of this work is to give extensions of inequalities (3) and (4) and establish their corresponding reversed versions. Moreover, the obtained results will be applied to improve Hao Z-C inequality [31] and Beckenbach-type inequality that is due to Wang [32]. The rest of this paper is organized as follows. In Section 2, we present extensions of (3) and (4) and establish their corresponding reversed versions. In Section 3, we apply the obtained results to improve Hao Z-C inequality and Beckenbachtype inequality that is due to Wang. Consequently, we obtain the refinement of arithmetic-geometric mean inequality. Finally, a brief summary is given in Section 4.

\section{Extension of Hu Ke's Inequality}

We begin this section with two lemmas, which will be used in the sequel.

Lemma 2.1. (e.g. [16], p. 12). Let $A_{k j}>0(j=1,2, \ldots, m, k=1,2, \ldots, n), \sum_{j=1}^{m} \frac{1}{\lambda_{j}}=1$. If $\lambda_{1}>0, \lambda_{j}<0(j=2,3, \ldots, m)$, then

$$
\sum_{k=1}^{n} \prod_{j=1}^{m} A_{k j} \geq \prod_{j=1}^{m}\left(\sum_{k=1}^{n} A_{k j}^{\lambda_{j}}\right)^{\frac{1}{\lambda_{j}}} .
$$

Lemma 2.2. [9]If $x>-1, \alpha>1$ or $\alpha<0$, then

$$
(1+x)^{\alpha} \geq 1+\alpha x .
$$

The inequality is reversed for $0<\alpha<1$.

Next, we give an extension of Hu Ke's inequality, as follows. 
Theorem 2.3. Let $A_{n j} \geq 0, \sum_{n} A_{n j}^{\lambda_{j}}<\infty(j=1,2, \ldots, k), \lambda_{1} \geq \lambda_{2} \geq \cdots \geq \lambda_{k}>0$, $\sum_{j=1}^{k} \frac{1}{\lambda_{j}}=1$, and let $1-e_{n}+e_{m} \geq 0, \Sigma_{n}\left|e_{n}\right|<\infty$. If $k$ is even, then

$$
\begin{aligned}
& \sum_{n} \prod_{j=1}^{k} A_{n j} \\
& \leq \prod_{j=1}^{\frac{k}{2}}\left\{\left(\sum_{n} A_{n(2 j-1)}^{\lambda_{2 j-1}}\right)^{\frac{1}{\lambda_{2 j-1}}-\frac{1}{\lambda_{2 j}}} \times\left[\left(\left(\sum_{n} A_{n(2 j-1)}^{\lambda_{2 j-1}}\right)\left(\sum_{n} A_{n(2 j)}^{\lambda_{2 j}}\right)\right)^{2}\right.\right. \\
& -\left(\left(\sum_{n} A_{n(2 j-1)}^{\lambda_{2 j-1}} e_{n}\right)\left(\sum_{n} A_{n(2 j)}^{\lambda_{2 j}}\right)\right. \\
& \left.\left.\left.-\left(\sum_{n} A_{n(2 j-1)}^{\lambda_{2 j-1}}\right)\left(\sum_{n} A_{n(2 j)}^{\lambda_{2 j}} e_{n}\right)\right)^{2}\right]^{\frac{1}{2 \lambda_{2 j}}}\right\} \text {. }
\end{aligned}
$$

If $k$ is odd, then

$$
\begin{gathered}
\sum_{n} \prod_{j=1}^{k} A_{n j} \leq\left(\sum_{n} A_{n k}^{\lambda_{k}}\right)^{\frac{1}{\lambda_{k}}} \times \prod_{j=1}^{\frac{k-1}{2}}\left\{\left(\sum_{n} A_{n(2 j-1)}^{\lambda_{2 j-1}}\right)^{\frac{1}{\lambda_{2 j-1}}-\frac{1}{\lambda_{2 j}}}\right. \\
\times\left[\left(\left(\sum_{n} A_{n(2 j-1)}^{\lambda_{2 j-1}}\right)\left(\sum_{n} A_{n(2 j)}^{\lambda_{2 j}}\right)\right)^{2}-\left(\left(\sum_{n} A_{n(2 j-1)}^{\lambda_{2 j-1}} e_{n}\right)\left(\sum_{n} A_{n(2 j)}^{\lambda_{2 j}}\right)\right.\right. \\
\left.\left.\left.-\left(\sum_{n} A_{n(2 j-1)}^{\lambda_{2 j-1}}\right)\left(\sum_{n} A_{n(2 j)}^{\lambda_{2 j}} e_{n}\right)\right)^{2}\right] \frac{1}{2 \lambda_{2 j}}\right\} .
\end{gathered}
$$

The integral form is as follows:

Theorem 2.4. Let $\lambda_{1} \geq \lambda_{2} \geq \cdots \geq \lambda_{k}>0, \sum_{j=1}^{k} \frac{1}{\lambda_{j}}=1$, let E be a measurable set, $F_{j}(x)$ be nonnegative measurable functions with $\int_{E} F_{j}^{\lambda_{j}}(x) d x<\infty$, and let $e(x)$ be a measurable function with $1-e(x)+e(y) \geq 0$. If $k$ is even, then

$$
\begin{gathered}
\int_{E} \prod_{j=1}^{k} F_{j}(x) \mathrm{d} x \leq \prod_{j=1}^{\frac{k}{2}}\left\{\left(\int_{E} F_{2 j-1}^{\lambda_{2 j-1}}(x) \mathrm{d} x\right)^{\frac{1}{\lambda_{2 j-1}}-\frac{1}{\lambda_{2 j}}}\right. \\
\times\left[\left(\int_{E} F_{2 j-1}^{\lambda_{2 j-1}}(x) \mathrm{d} x \int_{E} F_{2 j}^{\lambda_{2 j}}(x) \mathrm{d} x\right)^{2}\right. \\
-\left(\int_{E} F_{2 j-1}^{\lambda_{2 j-1}}(x) e(x) \mathrm{d} x \int_{E} F_{2 j}^{\lambda_{2 j}}(x) \mathrm{d} x\right. \\
\left.\left.\left.-\int_{E} F_{2 j-1}^{\lambda_{2 j-1}}(x) \mathrm{d} x \int_{E} F_{2 j}^{\lambda_{2 j}}(x) e(x) \mathrm{d} x\right)^{2}\right]^{\frac{1}{2 \lambda_{2 j}}}\right\} .
\end{gathered}
$$


If $k$ is odd, then

$$
\begin{gathered}
\int_{E} \prod_{j=1}^{k} F_{j}(x) \mathrm{d} x \leq\left(\int_{E} F_{k}^{\lambda_{k}}(x) \mathrm{d} x\right)^{\frac{1}{\lambda_{k}}} \times \prod_{j=1}^{\frac{k-1}{2}}\left\{\left(\int_{E} F_{2 j-1}^{\lambda_{2 j-1}}(x) \mathrm{d} x\right)^{\frac{1}{\lambda_{2 j-1}}-\frac{1}{\lambda_{2 j}}}\right. \\
\times\left[\left(\int_{E} F_{2 j-1}^{\lambda_{2 j-1}}(x) \mathrm{d} x \int_{E} F_{2 j}^{\lambda_{2 j}}(x) \mathrm{d} x\right)^{2}\right. \\
-\left(\int_{E} F_{2 j-1}^{\lambda_{2 j-1}}(x) e(x) \mathrm{d} x \int_{E} F_{2 j}^{\lambda_{2 j}}(x) \mathrm{d} x\right. \\
\left.\left.\left.-\int_{E} F_{2 j-1}^{\lambda_{2 j-1}}(x) \mathrm{d} x \int_{E} F_{2 j}^{\lambda_{2 j}}(x) e(x) \mathrm{d} x\right)^{2}\right]^{\frac{1}{2 \lambda_{2 j}}}\right\}
\end{gathered}
$$

Proof. We need to prove only Theorem 2.3. The proof of Theorem 2.4 is similar. A simple calculation gives

$$
\begin{aligned}
& \sum_{n}\left(\prod_{j=1}^{k} A_{n j}\right) \sum_{m}\left(\prod_{i=1}^{k} A_{m i}\right)\left(1-e_{n}+e_{m}\right) \\
& =\sum_{n} \sum_{m}\left(\prod_{j=1}^{k} A_{n j}\right)\left(\prod_{i=1}^{k} A_{m i}\right)-\sum_{n} \sum_{m}\left(\prod_{j=1}^{k} A_{n j}\right)\left(\prod_{i=1}^{k} A_{m i}\right) e_{n} \\
& \quad+\sum_{n} \sum_{m}\left(\prod_{j=1}^{k} A_{n j}\right)\left(\prod_{i=1}^{k} A_{m i}\right) e_{m} \\
& =\left(\sum_{n} \prod_{j=1}^{k} A_{n j}\right)^{2} .
\end{aligned}
$$

Case (I). When $k$ is even, by the inequality (2), we have

$$
\begin{aligned}
\sum_{n} & \left(\prod_{j=1}^{k} A_{n j}\right) \sum_{m}\left(\prod_{i=1}^{k} A_{m i}\right)\left(1-e_{n}+e_{m}\right) \\
= & \sum_{n}\left(\prod_{j=1}^{k} A_{n j}\right) \sum_{m} \prod_{i=1}^{k} A_{m i}\left(1-e_{n}+e_{m}\right)^{\frac{1}{\lambda_{i}}} \\
\leq & \sum_{n}\left(\prod_{j=1}^{k} A_{n j}\right)\left[\prod_{i=1}^{k}\left(\sum_{m} A_{m i}^{\lambda_{i}}\left(1-e_{n}+e_{m}\right)\right)^{\frac{1}{\lambda_{i}}}\right] \\
= & \sum_{n}\left\{\prod _ { j = 1 } ^ { \frac { k } { 2 } } \left[\left(A_{n(2 j-1)}^{\lambda_{2 j-1}} \sum_{m} A_{m(2 j-1)}^{\lambda_{2 j-1}}\left(1-e_{n}+e_{m}\right)\right)^{\frac{1}{\lambda_{2 j-1}}-\frac{1}{\lambda_{2 j}}}\right.\right. \\
& \times\left(A_{n(2 j-1)}^{\lambda_{2 j-1}} \sum_{m} A_{m(2 j)}^{\lambda_{2 j}}\left(1-e_{n}+e_{m}\right)\right)^{\frac{1}{\lambda_{2 j}}} \\
& \left.\left.\times\left(A_{n(2 j)}^{\lambda_{2 j}} \sum_{m} A_{m(2 j-1)}^{\lambda_{2 j-1}}\left(1-e_{n}+e_{m}\right)\right)^{\frac{1}{\lambda_{2 j}}}\right]\right\}
\end{aligned}
$$


Consequently, according to $\left(\frac{1}{\lambda_{1}}-\frac{1}{\lambda_{2}}\right)+\frac{1}{\lambda_{2}}+\frac{1}{\lambda_{2}}+\left(\frac{1}{\lambda_{3}}-\frac{1}{\lambda_{4}}\right)+\frac{1}{\lambda_{4}}+\frac{1}{\lambda_{4}}+\cdots+\left(\frac{1}{\lambda_{k-1}}-\frac{1}{\lambda_{k}}\right)+\frac{1}{\lambda_{k}}+\frac{1}{\lambda_{k}}=1$, by using the inequality (2) on the right side of (12), we observe that

$$
\begin{aligned}
& \sum_{n}\left(\prod_{j=1}^{k} A_{n j}\right) \sum_{m}\left(\prod_{i=1}^{k} A_{m i}\right)\left(1-e_{n}+e_{m}\right) \\
& \leq \prod_{j=1}^{\frac{k}{2}}\left[\left(\sum_{n} A_{n(2 j-1)}^{\lambda_{2 j-1}} \sum_{m} A_{m(2 j-1)}^{\lambda_{2 j-1}}\left(1-e_{n}+e_{m}\right)\right)^{\frac{1}{\lambda_{2 j-1}}-\frac{1}{\lambda_{2 j}}}\right. \\
& \times\left(\sum_{n} A_{n(2 j-1)}^{\lambda_{2 j-1}} \sum_{m} A_{m(2 j)}^{\lambda_{2 j}}\left(1-e_{n}+e_{m}\right)\right)^{\frac{1}{\lambda_{2 j}}} \\
& \left.\times\left(\sum_{n} A_{n(2 j)}^{\lambda_{2 j}} \sum_{m} A_{m(2 j-1)}^{\lambda_{2 j-1}}\left(1-e_{n}+e_{m}\right)\right)^{\frac{1}{\lambda_{2 j}}}\right] \\
& =\prod_{j=1}^{\frac{k}{2}}\left\{\left(\sum_{n} A_{n(2 j-1)}^{\lambda_{2 j-1}}\right)^{\frac{2}{\lambda_{2 j-1}}-\frac{2}{\lambda_{2 j}}} \times\left[\left(\sum_{n} \sum_{m} A_{n(2 j-1)}^{\lambda_{2 j-1}} A_{m(2 j)}^{\lambda_{2 j}}\left(1-e_{n}+e_{m}\right)\right)\right.\right. \\
& \left.\left.\times\left(\sum_{n} \sum_{m} A_{n(2 j)}^{\lambda_{2 j}} A_{m(2 j-1)}^{\lambda_{2 j-1}}\left(1-e_{n}+e_{m}\right)\right)\right]^{\frac{1}{\lambda_{2 j}}}\right\} \\
& =\prod_{j=1}^{\frac{k}{2}}\left\{\left(\sum_{n} A_{n(2 j-1)}^{\lambda_{2 j-1}}\right)^{\frac{2}{\lambda_{2 j-1}}-\frac{2}{\lambda_{2 j}}} \times\left[\left(\sum_{n} A_{n(2 j-1)}^{\lambda_{2 j-1}} \sum_{m} A_{m(2 j)}^{\lambda_{2 j}}\right.\right.\right. \\
& \left.-\sum_{n} A_{n(2 j-1)}^{\lambda_{2 j-1}} e_{n} \sum_{m} A_{m(2 j)}^{\lambda_{2 j}}+\sum_{n} A_{n(2 j-1)}^{\lambda_{2 j-1}} \sum_{m} A_{m(2 j)}^{\lambda_{2 j}} e_{m}\right) \\
& \times\left(\sum_{n} A_{n(2 j)}^{\lambda_{2 j}} \sum_{m} A_{m(2 j-1)}^{\lambda_{2 j-1}}-\sum_{n} A_{n(2 j)}^{\lambda_{2 j}} e_{n} \sum_{m} A_{m(2 j-1)}^{\lambda_{2 j-1}}\right. \\
& \left.\left.\left.+\sum_{n} A_{n(2 j)}^{\lambda_{2 j}} \sum_{m} A_{m(2 j-1)}^{\lambda_{2 j-1}} e_{m}\right)\right]^{\frac{1}{\lambda_{2 j}}}\right\} \\
& =\prod_{j=1}^{\frac{k}{2}}\left\{\left(\sum_{n} A_{n(2 j-1)}^{\lambda_{2 j-1}}\right)^{\frac{2}{\lambda_{2 j-1}}-\frac{2}{\lambda_{2 j}}} \times\left[\left(\left(\sum_{n} A_{n(2 j-1)}^{\lambda_{2 j-1}}\right)\left(\sum_{n} A_{n(2 j)}^{\lambda_{2 j}}\right)\right)^{2}\right.\right. \\
& -\left(\left(\sum_{n} A_{n(2 j-1)}^{\lambda_{2 j-1}} e_{n}\right)\left(\sum_{n} A_{n(2 j)}^{\lambda_{2 j}}\right)\right. \\
& \left.\left.\left.-\left(\sum_{n} A_{n(2 j-1)}^{\lambda_{2 j-1}}\right)\left(\sum_{n} A_{n(2 j)}^{\lambda_{2 j}} e_{n}\right)\right)^{2}\right]^{\frac{1}{\lambda_{2 j}}}\right\} .
\end{aligned}
$$

Combining inequalities (11) and (13) leads to inequality (7) immediately.

Case (II). When $k$ is odd, by the same method as in the above case (I), we have the inequality (8). The proof of Theorem 2.3 is complete.

To illustrate the significance of the introduction of the sequence $\left(e_{n}\right)_{n=1}^{\infty}$, let us sketch an example as follows.

Example 2.5. Let $\lambda_{j}=\frac{1}{2 N}, j=1,2, \ldots, 2 N, n=1,2, \ldots, 2 N, N \geq 2$, let $A_{n j}=\left\{\begin{array}{ll}1 \text { if } j=1, n=1,2, \ldots, 2 N \\ 1 & \text { if } n=j \\ 0 & \text { otherwise }\end{array}\right.$, and let $e_{n}=\left\{\begin{array}{l}0 \text { if } n \text { even } \\ 1 \text { if } n \text { odd }\end{array}\right.$. Then from the generalized 
Hölder inequality (2), we obtain $0 \leq(2 N) \frac{1}{2 N}$. However, from Theorem 2.3, we obtain $0 \leq 0$

Corollary 2.6. Let $A_{n j}, \lambda_{j}, e_{n}$ be as in Theorem 2.3, and let $\sum_{n} A_{n j}^{\lambda_{j}} \neq 0$. Then, the following inequality holds:

$$
\begin{aligned}
\sum_{n} & \prod_{j=1}^{k} A_{n j} \leq\left[\prod_{j=1}^{k}\left(\sum_{n} A_{n j}^{\lambda_{j}}\right)^{\frac{1}{\lambda_{j}}}\right] \\
& \times\left\{\prod_{j=1}^{\rho(k)}\left[1-\frac{1}{2 \lambda_{2 j}}\left(\frac{\sum_{n} A_{n(2 j-1)}^{\lambda_{2 j-1}} e_{n}}{\sum_{n} A_{n(2 j-1)}^{\lambda_{2 j-1}}}-\frac{\sum_{n} A_{n(2 j)}^{\lambda_{2 j}} e_{n}}{\sum_{n} A_{n(2 j)}^{\lambda_{2 j}}}\right)^{2}\right]\right\},
\end{aligned}
$$

where $\rho(k)=\left\{\begin{array}{cc}\frac{k}{2} \quad \text { if } k \text { even } \\ \frac{k-1}{2} \text { if } k \text { odd }\end{array}\right.$.

Corollary 2.7. Let $F_{j}(x), \lambda_{j}, e(x)$ be as in Theorem 2.4, and let $\int_{E} F_{j}^{\lambda_{j}}(x) \mathrm{d} x \neq 0$. Then, the following inequality holds:

$$
\begin{aligned}
& \int_{E} \prod_{j=1}^{k} F_{j}(x) \mathrm{d} x \leq\left[\prod_{j=1}^{k}\left(\int_{E} F_{j}^{\lambda_{j}}(x) \mathrm{d} x\right)^{\frac{1}{\lambda_{j}}}\right] \\
& \quad \times\left\{\prod_{j=1}^{\rho(k)}\left[1-\frac{1}{2 \lambda_{2 j}}\left(\frac{\int_{E} F_{2 j-1}^{\lambda_{2 j-1}}(x) e(x) \mathrm{d} x}{\int_{E} F_{2 j-1}^{\lambda_{2 j-1}}(x) \mathrm{d} x}-\frac{\int_{E} F_{2 j}^{\lambda_{2 j}}(x) e(x) \mathrm{d} x}{\int_{E} F_{2 j}^{\lambda_{2 j}}(x) \mathrm{d} x}\right)^{2}\right]\right\},
\end{aligned}
$$

where $\rho(k)=\left\{\begin{array}{cl}\frac{k}{2} \quad \text { if } k \text { even } \\ \frac{k-1}{2} \text { if } k \text { odd }\end{array}\right.$.

Proof. We need to prove only Corollary 2.6. The proof of Corollary 2.7 is similar. From inequalities (7) and (8), we obtain

$$
\begin{aligned}
& \sum_{n} \prod_{j=1}^{k} A_{n j} \leq\left[\prod_{j=1}^{k}\left(\sum_{n} A_{n j}^{\lambda_{j}}\right)^{\frac{1}{\lambda_{j}}}\right] \\
& \quad \times\left\{\prod_{j=1}^{\rho(k)}\left[1-\left(\frac{\sum_{n} A_{n(2 j-1)}^{\lambda_{2 j-1}} e_{n}}{\sum_{n} A_{n(2 j-1)}^{\lambda_{2 j-1}}}-\frac{\sum_{n} A_{n(2 j)}^{\lambda_{2 j}} e_{n}}{\sum_{n} A_{n(2 j)}^{\lambda_{2 j}}}\right)^{2}\right]^{\frac{1}{2 \lambda_{2 j}}}\right\} .
\end{aligned}
$$

Furthermore, performing some simple computations, we have

$$
\left|\frac{\sum_{n} A_{n(2 j-1)}^{\lambda_{2 j-1}} e_{n}}{\sum_{n} A_{n(2 j-1)}^{\lambda_{2 j-1}}}-\frac{\sum_{n} A_{n(2 j)}^{\lambda_{2 j}} e_{n}}{\sum_{n} A_{n(2 j)}^{\lambda_{2 j}}}\right|<1
$$

Consequently, from Lemma 2.2 and the inequalities (16) and (17), we have the desired inequality (14). The proof of Corollary 2.6 is complete.

It is clear that inequalities (7), (14) and (16) are sharper than the inequality (2).

Now, we present the following reversed versions of inequalities (7), (8), (9) and (10). 
Theorem 2.8. Let $A_{r j}>0,(r=1,2, \ldots, n, j=1,2, \ldots, m), \sum_{j=1}^{m} \frac{1}{\lambda_{j}}=1$, and let $1-e_{r}+e_{s}$ $\geq 0(s=1,2, \ldots, n)$. If $\lambda_{1}>0, \lambda_{j}<0(j=2,3, \ldots, m)$, then

$$
\begin{gathered}
\sum_{r=1}^{n} \prod_{j=1}^{m} A_{r j} \geq\left(\sum_{r=1}^{n} A_{r 1}^{\lambda_{1}}\right)^{\frac{1}{\lambda_{1}}-\sum_{j=2}^{m} \frac{1}{\lambda_{j}}} \prod_{j=2}^{m}\left\{\left[\left(\sum_{r=1}^{n} A_{r 1}^{\lambda_{1}}\right)\left(\sum_{r=1}^{n} A_{r j}^{\lambda_{j}}\right)\right]^{2}\right. \\
\left.-\left[\left(\sum_{r=1}^{n} A_{r 1}^{\lambda_{1}} e_{r}\right)\left(\sum_{r=1}^{n} A_{r j}^{\lambda_{j}}\right)-\left(\sum_{r=1}^{n} A_{r 1}^{\lambda_{1}}\right)\left(\sum_{r=1}^{n} A_{r j}^{\lambda_{j}} e_{r}\right)\right]^{2}\right\}^{\frac{1}{2 \lambda_{j}}} .
\end{gathered}
$$

The integral form is as follows:

Theorem 2.9. Let $F_{j}(x)$ be nonnegative integrable functions on $[a, b]$ such that $\int_{a}^{b} F_{j}^{\lambda_{j}}(x) \mathrm{d}$ xexist, let $1-e(x)+e(y) \geq 0$ for all $x, y \in[a, b]$, and $\int_{a}^{b} e(x) \mathrm{d} x<\infty$, and let $\sum_{j=1}^{m} \frac{1}{\lambda_{j}}=1$. If $\lambda_{1}>0, \lambda_{j}<0(j=2,3, \ldots, m)$, then

$$
\begin{aligned}
\int_{a}^{b} \prod_{j=1}^{m} F_{j}(x) \mathrm{d} x \geq\left(\int_{a}^{b} F_{1}^{\lambda_{1}}(x) \mathrm{d} x\right)^{\frac{1}{\lambda_{1}}-\sum_{j=2}^{m} \frac{1}{\lambda_{j}}} \\
\times \prod_{j=2}^{m}\left[\left(\int_{a}^{b} F_{1}^{\lambda_{1}}(x) \mathrm{d} x \int_{a}^{b} F_{j}^{\lambda_{j}}(x) \mathrm{d} x\right)^{2}\right. \\
-\left(\int_{a}^{b} F_{1}^{\lambda_{1}}(x) e(x) \mathrm{d} x \int_{a}^{b} F_{j}^{\lambda_{j}}(x) \mathrm{d} x\right. \\
\left.\left.-\int_{a}^{b} F_{1}^{\lambda_{1}}(x) \mathrm{d} x \int_{a}^{b} F_{j}^{\lambda_{j}}(x) e(x) \mathrm{d} x\right)^{2}\right]^{\frac{1}{2 \lambda_{j}}} .
\end{aligned}
$$

Proof. We need to prove only Theorem 2.8. The proof of Theorem 2.9 is similar. By the inequality (5), we have

$$
\begin{aligned}
\sum_{s=1}^{n} & \left(\prod_{i=1}^{m} A_{s i}\right) \sum_{r=1}^{n}\left(\prod_{j=1}^{m} A_{r j}\right)\left(1-e_{r}+e_{s}\right) \\
= & \sum_{s=1}^{n}\left(\prod_{i=1}^{m} A_{s i}\right) \sum_{r=1}^{n} \prod_{j=1}^{m} A_{r j}\left(1-e_{r}+e_{s}\right)^{\frac{1}{\lambda_{j}}} \\
\geq & \sum_{s=1}^{n}\left(\prod_{i=1}^{m} A_{s i}\right)\left[\prod_{j=1}^{m}\left(\sum_{r=1}^{n} A_{r j}^{\lambda_{j}}\left(1-e_{r}+e_{s}\right)\right)^{\frac{1}{\lambda_{j}}}\right] \\
= & \sum_{s=1}^{n}\left\{\left(A_{s 1}^{\lambda_{1}} \sum_{r=1}^{n} A_{r 1}^{\lambda_{1}}\left(1-e_{r}+e_{s}\right)\right)^{\frac{1}{\lambda_{1}}-\sum_{j=2}^{m} \frac{1}{\lambda_{j}}}\right. \\
& \times\left[\prod_{j=2}^{m}\left(A_{s 1}^{\lambda_{1}} \sum_{r=1}^{n} A_{r j}^{\lambda_{j}}\left(1-e_{r}+e_{s}\right)\right)^{\frac{1}{\lambda_{j}}}\right] \\
& \left.\times\left[\prod_{j=2}^{m}\left(A_{s j}^{\lambda_{j}} \sum_{r=1}^{n} A_{r 1}^{\lambda_{1}}\left(1-e_{r}+e_{s}\right)\right)^{\frac{1}{\lambda_{j}}}\right]\right\}
\end{aligned}
$$


Consequently, $\left(\frac{1}{\lambda_{1}}-\sum_{j=2}^{m} \frac{1}{\lambda_{j}}\right)+\frac{1}{\lambda_{2}}+\frac{1}{\lambda_{3}}+\cdots+\frac{1}{\lambda_{m}}+\frac{1}{\lambda_{2}}+\frac{1}{\lambda_{3}}+\cdots+\frac{1}{\lambda_{m}}=1$, by using the inequality (5) on the right side of (20), we observe that

$$
\begin{aligned}
& \sum_{s=1}^{n}\left(\prod_{i=1}^{m} A_{s i}\right) \sum_{r=1}^{n}\left(\prod_{j=1}^{m} A_{r j}\right)\left(1-e_{r}+e_{s}\right) \\
& \geq\left(\sum_{s=1}^{n} \sum_{r=1}^{n} A_{s 1}^{\lambda_{1}} A_{r 1}^{\lambda_{1}}\left(1-e_{r}+e_{s}\right)\right)^{\frac{1}{\lambda_{1}}-\sum_{j=2}^{m} \frac{1}{\lambda_{j}}} \\
& \times\left[\prod_{j=2}^{m}\left(\sum_{s=1}^{n} \sum_{r=1}^{n} A_{s 1}^{\lambda_{1}} A_{r j}^{\lambda_{j}}\left(1-e_{r}+e_{s}\right)\right)^{\frac{1}{\lambda_{j}}}\right] \\
& \times\left[\prod_{j=2}^{m}\left(\sum_{s=1}^{n} \sum_{r=1}^{n} A_{s j}^{\lambda_{j}} A_{r 1}^{\lambda_{1}}\left(1-e_{r}+e_{s}\right)\right)^{\frac{1}{\lambda_{j}}}\right] \\
& =\left(\sum_{r=1}^{n} A_{r 1}^{\lambda_{1}}\right)^{\frac{2}{\lambda_{1}}-\sum_{j=2}^{m} \frac{2}{\lambda_{j}}} \times\left\{\prod _ { j = 2 } ^ { m } \left[\left(\sum_{s=1}^{n} \sum_{r=1}^{n} A_{s 1}^{\lambda_{1}} A_{r j}^{\lambda_{j}}\left(1-e_{r}+e_{s}\right)\right)\right.\right. \\
& \left.\left.\times\left(\sum_{s=1}^{n} \sum_{r=1}^{n} A_{s j}^{\lambda_{j}} A_{r 1}^{\lambda_{1}}\left(1-e_{r}+e_{s}\right)\right)\right]^{\frac{1}{\lambda_{j}}}\right\} \\
& =\left(\sum_{r=1}^{n} A_{r 1}^{\lambda_{1}}\right)^{\frac{2}{\lambda_{1}}-\sum_{j=2}^{m} \frac{2}{\lambda_{j}}} \times\left\{\prod _ { j = 2 } ^ { m } \left[\left(\sum_{s=1}^{n} A_{s 1}^{\lambda_{1}} \sum_{r=1}^{n} A_{r j}^{\lambda_{j}}\right.\right.\right. \\
& \left.-\sum_{s=1}^{n} A_{s 1}^{\lambda_{1}} \sum_{r=1}^{n} A_{r j}^{\lambda_{j}} e_{r}+\sum_{s=1}^{n} A_{s 1}^{\lambda_{1}} e_{s} \sum_{r=1}^{n} A_{r j}^{\lambda_{j}}\right) \\
& \left.\left.\times\left(\sum_{s=1}^{n} A_{s j}^{\lambda_{j}} \sum_{r=1}^{n} A_{r 1}^{\lambda_{1}}-\sum_{s=1}^{n} A_{s j}^{\lambda_{j}} \sum_{r=1}^{n} A_{r 1}^{\lambda_{1}} e_{r}+\sum_{s=1}^{n} A_{s j}^{\lambda_{j}} e_{s} \sum_{r=1}^{n} A_{r 1}^{\lambda_{1}}\right)\right]^{\frac{1}{\lambda_{j}}}\right\} \\
& =\left(\sum_{r=1}^{n} A_{r 1}^{\lambda_{1}}\right)^{\frac{2}{\lambda_{1}}-\sum_{j=2}^{m} \frac{2}{\lambda_{j}}} \times\left\{\prod _ { j = 2 } ^ { m } \left[\left(\left(\sum_{r=1}^{n} A_{r 1}^{\lambda_{1}}\right)\left(\sum_{r=1}^{n} A_{r j}^{\lambda_{j}}\right)\right)^{2}\right.\right. \\
& \text { - } \left.\left.\left(\left(\sum_{r=1}^{n} A_{r 1}^{\lambda_{1}}\right)\left(\sum_{r=1}^{n} A_{r j}^{\lambda_{j}} e_{r}\right)-\left(\sum_{r=1}^{n} A_{r 1}^{\lambda_{1}} e_{r}\right)\left(\sum_{r=1}^{n} A_{r j}^{\lambda_{j}}\right)\right)^{2}\right]^{\frac{1}{\lambda_{j}}}\right\} .
\end{aligned}
$$

Combining inequalities (11) and (21) leads to inequality (18) immediately. The proof of Theorem 2.8 is complete. $\square$

Corollary 2.10. Let $A_{r j}, \lambda_{j}, e_{r}$ be as in Theorem 2.8, and let $\sum_{r=1}^{n} A_{r j}^{\lambda_{j}} \neq 0$. Then

$$
\begin{aligned}
& \sum_{r=1}^{n} \prod_{j=1}^{m} A_{r j} \\
& \geq\left[\prod_{j=1}^{m}\left(\sum_{r=1}^{n} A_{r j}^{\lambda_{j}}\right)^{\frac{1}{\lambda_{j}}}\right]\left\{\prod_{j=2}^{m}\left[1-\frac{1}{2 \lambda_{j}}\left(\frac{\sum_{r=1}^{n} A_{r 1}^{\lambda_{1}} e_{r}}{\sum_{r=1}^{n} A_{r 1}^{\lambda_{1}}}-\frac{\sum_{r=1}^{n} A_{r j}^{\lambda_{j}} e_{r}}{\sum_{r=1}^{n} A_{r j}^{\lambda_{j}}}\right)^{2}\right]\right\} .
\end{aligned}
$$


Corollary 2.11. Let $F_{j}(x), \lambda_{j}, e(x)$ be as in Theorem 2.9, and let $\int_{a}^{b} F_{j}^{\lambda_{j}}(x) \mathrm{d} x \neq 0$. Then

$$
\begin{aligned}
& \int_{a}^{b} \prod_{j=1}^{m} F_{j}(x) \mathrm{d} x \geq\left[\prod_{j=1}^{m}\left(\int_{a}^{b} F_{j}^{\lambda_{j}}(x) \mathrm{d} x\right)^{\frac{1}{\lambda_{j}}}\right] \\
& \quad \times\left\{\prod_{j=2}^{m}\left[1-\frac{1}{2 \lambda_{j}}\left(\frac{\int_{a}^{b} F_{1}^{\lambda_{1}}(x) e(x) \mathrm{d} x}{\int_{a}^{b} F_{1}^{\lambda_{1}}(x) \mathrm{d} x}-\frac{\int_{a}^{b} F_{j}^{\lambda_{j}}(x) e(x) \mathrm{d} x}{\int_{a}^{b} F_{j}^{\lambda_{j}}(x) \mathrm{d} x}\right)^{2}\right]\right\} .
\end{aligned}
$$

Proof. Making similar arguments as in the proof of Corollary 2.6, we have the desired inequalities (22) and (23).

It is clear that inequalities (18) and (22) are sharper than the generalized Hölder inequality (5).

Now, we give here some direct consequences from Theorem 2.8 and Theorem 2.9. Putting $m=2$ in (18) and (19), respectively, we obtain the following corollaries.

Corollary 2.12. Let $A_{r 1}, A_{r 2}, \lambda_{1}, \lambda_{2}, e_{r}$ be as in Theorem 2.8. Then, the following reversed version of $\mathrm{Hu}$ Ke's inequality (3) holds:

$$
\begin{aligned}
& \sum_{r=1}^{n} A_{r 1} A_{r 2} \geq\left(\sum_{r=1}^{n} A_{r 1}^{\lambda_{1}}\right)^{\frac{1}{\lambda_{1}}-\frac{1}{\lambda_{2}}}\left[\left(\sum_{r=1}^{n} A_{r 1}^{\lambda_{1}}\right)\left(\sum_{r=1}^{n} A_{r 2}^{\lambda_{2}}\right)\right]^{2} \\
& \left.-\left[\left(\sum_{r=1}^{n} A_{r 1}^{\lambda_{1}} e_{r}\right)\left(\sum_{r=1}^{n} A_{r 2}^{\lambda_{2}}\right)-\left(\sum_{r=1}^{n} A_{r 1}^{\lambda_{1}}\right)\left(\sum_{r=1}^{n} A_{r 2}^{\lambda_{2}} e_{r}\right)\right]^{2}\right\}^{\frac{1}{2 \lambda_{2}}} .
\end{aligned}
$$

Corollary 2.13. Let $F_{1}(x), F_{2}(x), \lambda_{1}, \lambda_{2}, e(x)$ be as in Theorem 2.9. Then, the following reversed version of $\mathrm{Hu} \mathrm{Ke}$ 's inequality (4) holds:

$$
\begin{aligned}
& \int_{a}^{b} F_{1}(x) F_{2}(x) \mathrm{d} x \\
& \geq\left(\int_{a}^{b} F_{1}^{\lambda_{1}}(x) \mathrm{d} x\right)^{\frac{1}{\lambda_{1}}-\frac{1}{\lambda_{2}}} \times\left[\left(\int_{a}^{b} F_{1}^{\lambda_{1}}(x) \mathrm{d} x \int_{a}^{b} F_{2}^{\lambda_{2}}(x) \mathrm{d} x\right)^{2}\right. \\
& -\left(\int_{a}^{b} F_{1}^{\lambda_{1}}(x) e(x) \mathrm{d} x \int_{a}^{b} F_{2}^{\lambda_{2}}(x) \mathrm{d} x\right. \\
& \left.\left.-\int_{a}^{b} F_{1}^{\lambda_{1}}(x) \mathrm{d} x \int_{a}^{b} F_{2}^{\lambda_{2}}(x) e(x) \mathrm{d} x\right)^{2}\right]^{\frac{1}{2 \lambda_{2}}}
\end{aligned}
$$

Example 2.14. Putting $e(x)=\frac{1}{2} \cos \frac{\pi(b-x)}{b-a}$ in (23), we obtain

$$
\begin{aligned}
& \int_{a}^{b} \prod_{j=1}^{m} F_{j}(x) \mathrm{d} x \geq\left[\prod_{j=1}^{m}\left(\int_{a}^{b} F_{j}^{\lambda_{j}}(x) \mathrm{d} x\right)^{\frac{1}{\lambda_{j}}}\right] \\
& \quad \times\left\{\prod_{j=2}^{m}\left[1-\frac{1}{8 \lambda_{j}}\left(\frac{\int_{a}^{b} F_{1}^{\lambda_{1}}(x) \cos \frac{\pi(b-x)}{b-a} \mathrm{~d} x}{\int_{a}^{b} F_{1}^{\lambda_{1}}(x) \mathrm{d} x}-\frac{\int_{a}^{b} F_{j}^{\lambda_{j}}(x) \cos \frac{\pi(b-x)}{b-a} \mathrm{~d} x}{\int_{a}^{b} F_{j}^{\lambda_{j}}(x) \mathrm{d} x}\right)^{2}\right]\right\},
\end{aligned}
$$


where $\lambda_{1}>0, \lambda_{j}<0(j=2,3, \ldots, m), \sum_{j=1}^{m} \frac{1}{\lambda_{j}}=1$.

\section{Applications}

In this section, we show some applications of our new inequalities. Firstly, we provide an application of the obtained results to improve Hao Z-C inequality, which is related to the generalized arithmetic-geometric mean inequality with weights. The generalized arithmetic-geometric mean inequality (e.g. [9]) states that if $a_{j}>0, \lambda_{j}>0(j=1,2, \ldots$, $k), p>0$ and $\sum_{j=1}^{k} \frac{1}{\lambda_{j}}=1$, then

$$
\prod_{j=1}^{k} a_{j}^{\frac{1}{\lambda_{j}}} \leq \sum_{j=1}^{k} \frac{a_{j}}{\lambda_{j}}
$$

The classical arithmetic-geometric mean inequality is one of the most important inequalities in analysis. This classical inequality has been widely studied by many authors, and it has motivated a large number of research papers involving different proofs, various generalizations and improvements (see e.g. [1,9,12,19,33] and references therein). In the year 1990, Hao Z-C in [31] established the following interesting inequality

$$
\prod_{j=1}^{k} a_{j}^{\frac{1}{\lambda_{j}}} \leq\left\{p \int_{0}^{\infty}\left[\prod_{j=1}^{k}\left(x+a_{j}\right)^{\frac{1}{\lambda_{j}}}\right]^{-p-1} \mathrm{~d} x\right\}^{-\frac{1}{p}} \leq \sum_{j=1}^{k} \frac{a_{j}}{\lambda_{j}}
$$

where $a_{j}>0, \lambda_{j}>0(j=1,2, \ldots, k), p>0$ and $\sum_{j=1}^{k} \frac{1}{\lambda_{j}}=1$. The above Hao Z-C inequality is refined by using Corollary 2.7 as follows:

Theorem 3.1. Let $a_{j}>0(j=1,2, \ldots, k), p>0$, let $\lambda_{1} \geq \lambda_{2} \geq \cdots \geq \lambda_{k}>0, \sum_{j=1}^{k} \frac{1}{\lambda_{j}}=1$, and let $1-e(x)+e(y) \geq 0, \int_{0}^{\infty} e(x) \mathrm{d} x<\infty$. Then

$$
\begin{gathered}
\prod_{j=1}^{k} a_{j}^{\frac{1}{\lambda_{j}}} \leq\left(\prod_{j=1}^{k} a_{j}^{\frac{1}{\lambda_{j}}}\right) \times\left[\prod_{j=1}^{\rho(k)}\left(1-\frac{1}{2 \lambda_{j}} R^{2}\left(x, e_{i} a_{j}, p\right)\right)\right]^{-\frac{1}{p}} \\
\leq\left\{p \int_{0}^{\infty}\left[\prod_{j=1}^{k}\left(x+a_{j}\right)^{\frac{1}{\lambda_{j}}}\right]^{-p-1} d x\right\}^{-\frac{1}{p}} \leq \sum_{j=1}^{k} \frac{a_{j}}{\lambda_{j}}, \\
\text { where } \rho(k)=\left\{\frac{k \frac{k}{2} \text { if } k \text { even }}{2} \text { if } k\right. \text { odd } \\
R\left(x, e ; a_{j}, p\right)=\frac{\int_{0}^{\infty}\left(x+a_{2 j-1}\right)^{-p-1} e(x) \mathrm{d} x}{\int_{0}^{\infty}\left(x+a_{2 j-1}\right)^{-p-1} \mathrm{~d} x}-\frac{\int_{0}^{\infty}\left(x+a_{2 j}\right)^{-p-1} e(x) \mathrm{d} x}{\int_{0}^{\infty}\left(x+a_{2 j}\right)^{-p-1} \mathrm{~d} x} .
\end{gathered}
$$


Proof. For $x \geq 0$, with a substitution $a_{j} \rightarrow x+a_{j}$ in (27), we have

$$
0<\prod_{j=1}^{k}\left(x+a_{j}\right)^{\frac{1}{\lambda_{j}}} \leq \sum_{j=1}^{k} \frac{x+a_{j}}{\lambda_{j}}=x+\sum_{j=1}^{k} \frac{a_{j}}{\lambda_{j}}
$$

Now, integrating both sides of (30) from 0 to $\infty$, we observe that

$$
\int_{0}^{\infty}\left(\prod_{j=1}^{k}\left(x+a_{j}\right)^{\frac{1}{\lambda_{j}}}\right)^{-p-1} \mathrm{~d} x \geq \int_{0}^{\infty}\left[x+\sum_{j=1}^{k} \frac{a_{j}}{\lambda_{j}}\right]^{-p-1} \mathrm{~d} x=\frac{1}{p}\left(\sum_{j=1}^{k} \frac{a_{j}}{\lambda_{j}}\right)^{-p}
$$

On the other hand, applying the inequality (15), we obtain

$$
\begin{aligned}
& \int_{0}^{\infty}\left[\prod_{j=1}^{k}\left(x+a_{j}\right)^{\frac{1}{\lambda_{j}}}\right]^{-p-1} \mathrm{~d} x=\int_{0}^{\infty} \prod_{j=1}^{k}\left[\left(x+a_{j}\right)^{-p-1}\right]^{\frac{1}{\lambda_{j}}} \mathrm{~d} x \\
& \leq\left[\prod_{j=1}^{k}\left(\int_{0}^{\infty}\left(x+a_{j}\right)^{-p-1} \mathrm{~d} x\right)^{\frac{1}{\lambda_{j}}}\right] \times\left[\prod_{j=1}^{\rho(k)}\left(1-\frac{1}{2 \lambda_{2 j}} R^{2}\left(x, e ; a_{j}, p\right)\right)\right] \\
& \quad=\left(\frac{1}{p} \prod_{j=1}^{k} a_{j}^{-\frac{p}{\lambda_{j}}}\right) \times\left[\prod_{j=1}^{\rho(k)}\left(1-\frac{1}{2 \lambda_{2 j}} R^{2}\left(x, e_{i} a_{j}, p\right)\right)\right] .
\end{aligned}
$$

Combining inequalities (32) and (31) yields inequality (29) immediately. The proof of Theorem 3.1 is complete.

From Theorem 3.1, we have the following Corollary.

Corollary 3.2. With notation as in Theorem 3.1, we have

$$
\prod_{j=1}^{k} a_{j}^{\frac{1}{\lambda_{j}}} \leq\left[\prod_{j=2}^{\rho(k)}\left(1-\frac{1}{2 \lambda_{j}} R^{2}\left(x, e ; a_{j}, p\right)\right)\right]^{\frac{1}{p}}\left(\sum_{j=1}^{k} \frac{a_{j}}{\lambda_{j}}\right) .
$$

It is clear that inequality (33) is sharper than the inequality (27).

Now, we give a sharpness of Beckenbach-type inequality from Corollary 2.10. The famous Beckenbach inequality [8] has been generalized and extended in several directions; see, e.g., [16]. In 1983, Wang [32] established the following Beckenbach-type inequality.

Theorem D. Let $f(x), g(x)$ be positive integrable functions defined on $[0, T]$, and let $\frac{1}{p}+\frac{1}{q}=1$. If $0<p<1$, then, for any positive numbers $a, b, c$, the inequality

$$
\frac{\left(a+c \int_{0}^{T} h^{p}(x) \mathrm{d} x\right)^{\frac{1}{p}}}{b+c \int_{0}^{T} h(x) g(x) \mathrm{d} x} \geq \frac{\left(a+c \int_{0}^{T} f^{p}(x) \mathrm{d} x\right)^{\frac{1}{p}}}{b+c \int_{0}^{T} f(x) g(x) \mathrm{d} x}
$$

holds, where $h(x)=\left(\frac{a g(x)}{b}\right)^{\frac{q}{p}}$. The sign of the inequality in (34) is reversed if $p>1$. 
Theorem 3.3. Let $f(x), g(x), e(x)$ be integrable functions defined on $[0, T]$, let $f(x), g(x)$ $>0,1-e(x)+e(y) \geq 0$ for all $x, y \in[0, T]$, and let $\frac{1}{p}+\frac{1}{q}=1$. If $0<p<1$, then, for any positive numbers $a, b, c$, the inequality

$$
\begin{aligned}
& \frac{\left(a+c \int_{0}^{T} h^{p}(x) \mathrm{d} x\right)^{\frac{1}{p}}}{b+c \int_{0}^{T} h(x) g(x) \mathrm{d} x} \geq \frac{\left(a+c \int_{0}^{T} f^{p}(x) \mathrm{d} x\right)^{\frac{1}{p}}}{b+c \int_{0}^{T} f(x) g(x) \mathrm{d} x} \\
& \quad \times\left[1-\frac{1}{2 q}\left(\frac{c \int_{0}^{T} f^{p}(x) \mathrm{d} x}{a+c \int_{0}^{T} f^{p}(x) \mathrm{d} x}-\frac{c \int_{0}^{T} g^{q}(x) \mathrm{d} x}{a^{-\frac{q}{p}} b^{q}+c \int_{0}^{T} g^{q}(x) \mathrm{d} x}\right)^{2}\right]
\end{aligned}
$$

holds, where $h(x)=\left(\frac{a g(x)}{b}\right)^{\frac{q}{p}}$

Proof. Performing some simple computations, we have

$$
\frac{\left(a+c \int_{0}^{T} h^{p}(x) \mathrm{d} x\right)^{\frac{1}{p}}}{b+c \int_{0}^{T} h(x) g(x) \mathrm{d} x}=\left(a^{-\frac{q}{p}} b^{q}+c \int_{0}^{T} g^{q}(x) \mathrm{d} x\right)^{-\frac{1}{q}} .
$$

On the other hand, putting $e_{1}=0, e_{2}=1, m=2$ in (22), from Corollary 2.10 we obtain

$$
\begin{aligned}
b+ & c \int_{0}^{T} f(x) g(x) \mathrm{d} x \geq b+c\left(\int_{0}^{T} f^{p}(x) \mathrm{d} x\right)^{\frac{1}{p}}\left(\int_{0}^{T} g^{q}(x) \mathrm{d} x\right)^{\frac{1}{q}} \\
& =a^{\frac{1}{p}}\left(b a^{-\frac{1}{p}}\right)+\left(c \int_{0}^{T} f^{p}(x) \mathrm{d} x\right)^{\frac{1}{p}}\left(c \int_{0}^{T} g^{q}(x) \mathrm{d} x\right)^{\frac{1}{q}} \\
\geq & \left(a+c \int_{0}^{T} f^{p}(x) \mathrm{d} x\right)^{\frac{1}{p}}\left(a-\frac{q}{p} b^{q}+c \int_{0}^{T} g^{q}(x) \mathrm{d} x\right)^{\frac{1}{q}} \\
& \times\left[1-\frac{1}{2 q}\left(\frac{c \int_{0}^{T} f^{p}(x) \mathrm{d} x}{a+c \int_{0}^{T} f^{p}(x) \mathrm{d} x}-\frac{c \int_{0}^{T} g^{q}(x) \mathrm{d} x}{a^{-\frac{q}{p}} b^{q}+c \int_{0}^{T} g^{q}(x) \mathrm{d} x}\right)^{2}\right],
\end{aligned}
$$

that is

$$
\begin{aligned}
& \left(a a^{-\frac{q}{p}} b^{q}+c \int_{0}^{T} g^{q}(x) \mathrm{d} x\right)^{-\frac{1}{q}} \geq \frac{\left(a+c \int_{0}^{T} f^{p}(x) \mathrm{d} x\right)^{\frac{1}{p}}}{b+c \int_{0}^{T} f(x) g(x) \mathrm{d} x} \\
& \times\left[1-\frac{1}{2 q}\left(\frac{c \int_{0}^{T} f^{p}(x) \mathrm{d} x}{a+c \int_{0}^{T} f^{p}(x) \mathrm{d} x}-\frac{c \int_{0}^{T} g^{q}(x) \mathrm{d} x}{a^{-\frac{q}{p}} b^{q}+c \int_{0}^{T} g^{q}(x) \mathrm{d} x}\right)^{2}\right] .
\end{aligned}
$$


Combining inequalities (36) and (38) yields inequality (35). The proof of Theorem 3.3 is complete.

\section{Conclusions}

The classical Hölder's inequality plays a very important role in both theory and applications. In this paper, we have presented an extension of $\mathrm{Hu}$ Ke's inequality, which is a sharp Hölder's inequality, and established their corresponding reversed versions. Moreover, we have improved Hao Z-C inequality and Beckenbach-type inequality by using the obtained results. Finally, we have obtained the refinement of arithmetic-geometric mean inequality. We think that our results will be useful for those areas in which inequalities (2) and (5) play a role. In the future research, we will continue to explore other applications of the obtained inequalities.

\section{Acknowledgements}

The author would like to express his sincere thanks to the anonymous referees for their making great efforts to improve this paper. This work was supported by the NNSF of China (Grant No. 61073121), and the Fundamental Research Funds for the Central Universities (No. 11ML65).

\section{Competing interests}

The author declares that they have no competing interests.

Received: 30 April 2011 Accepted: 6 October 2011 Published: 6 October 2011

\section{References}

1. Mitrinović, DS, Vasić, PM: Analytic Inequalities. Springer, New York (1970)

2. Agarwal, RP: Difference Equations and Inequalities. Marcel Dekker, New York, 2 (2000)

3. Agarwal, RP: Inequalities and Applications. World Scientific, Singapore (1994)

4. Agarwal, RP, Barnett, NS, Cerone, P, Dragomir, SS: A survey on some inequalities for expectation and variance. Comput Math Appl. 49(2-3), 429-480 (2005). doi:10.1016/j.camwa.2002.11.026

5. Agarwal, RP, Dragomir, SS: The property of supermultiplicity for some classical inequalities and applications. Comput Math Appl. 35(6), 105-118 (1998). doi:10.1016/S0898-1221(98)00023-6

6. Agarwal, RP, Pang, PYH: Discrete opial-type inequalities involving higher order partial differences. Nonlinear Anal Theor. 27(4):429-454 (1996). doi:10.1016/0362-546X(95)00020-V

7. Aldaz, JM: A stability version of Hölder's inequality. J Math Anal Appl. 343, 842-845 (2008). doi:10.1016/j. jmaa.2008.01.104

8. Beckenbach, EF: On Hölder's inequality. J Math Anal Appl. 15, 21-29 (1966). doi:10.1016/0022-247X(66)90133-8

9. Beckenbach, EF, Bellman, R: Inequalities. Springer, Berlin (1983)

10. Bourin, J-C, Lee, E-Y, Fujii, M, Seo, Y: A matrix reverse Hölder inequality. Linear Algebra Appl. 431, 2154-2159 (2009). doi:10.1016/j.laa.2009.07.010

11. Fujii, M, Lee, E-Y, Seo, Y: A difference counterpart to a matrix Hölder inequality. Linear Algebra Appl. 432, 2565-2571 (2010). doi:10.1016/j.laa.2009.12.002

12. Hardy, G, Littlewood, JE, Pólya, G: Inequalities. Cambridge University Press, UK, 2 (1952)

13. Hu, K: On an inequality and its applications. Sci Sinica. 24, 1047-1055 (1981)

14. Jang, L-C: A note on Hölder type inequality for the fermionic p-adic invariant q-integral. J Inequal Appl (2009) 2009, 5 (2009). Article ID 357349

15. Kwon, EG, Bae, EK: On a continuous form of Hölder inequality. J Math Anal Appl. 343, 585-592 (2008)

16. Kuang, J: Applied Inequalities. Shandong Science and Technology Press, Jinan, 4 (2010)

17. Liu, B: Inequalities and convergence concepts of fuzzy and rough variables. Fuzzy Optim Decis Ma. 2(2):87-100 (2003). doi:10.1023/A:1023491000011

18. Liu, B: Uncertainty Theory. Springer, Berlin, 2 (2007)

19. Mitrinović, DS, Pečarić, JE, Fink, AM: Classical and New Inequalities in Analysis. Dordrecht:Kluwer (1993)

20. Mond, B, Pečarić, JE: On converses of Hölder and Beckenbach inequalities. J Math Anal Appl. 196, 795-799 (1995). doi:10.1006/jmaa.1995.1443

21. Neugebauer, CJ: Some inequalities related to Hölder's inequality. Proc Am Math Soc. 82, 560-564 (1981)

22. Pang, PYH, Agarwal, RP: On an Opial type inequality due to Fink. J Math Anal Appl. 196(2), 748-753 (1995). doi:10.1006/ jmaa.1995.1438

23. Pang, PYH, Agarwal, RP: On an integral inequality and its discrete analogue. J Math Anal Appl. 194(2), 569-577 (1995). doi:10.1006/jmaa.1995.1318

24. Singh, RP, Rajeev, Kumar, Tuteja, RK: Application of Hölder's inequality in information theory. Inform Sci. 152, 145-154 (2003)

25. Tian, J: Inequalities and mathematical properties of uncertain variables. Fuzzy Optim Decis Ma. (2011)

26. Tian, J, Zhang, Z, Tian, D: Moment estimation inequalities based on $g_{\lambda}$ random variable on Sugeno measure space. J Inequal Appl 2010, 10 (2010). Article ID 290124 
27. Wang, R-S: Some inequalities and convergence theorems for Choquet integrals. J Appl Math Comput. 35(1-2):305-321 (2011). doi:10.1007/s12190-009-0358-y

28. Wu, L, Sun, J, Ye, X, Zhu, L: Hölder type inequality for Sugeno integral. Fuzzy Set Syst. 161, 2337-2347 (2010). doi:10.1016/j.fss.2010.04.017

29. Yang, W: A functional generalization of diamond-a integral Hölder's nequality on time scales. Appl Math Lett. 23 1208-1212 (2010). doi:10.1016/j.aml.2010.05.013

30. Zhu, Y, Liu, B: Some inequalities of random fuzzy variables with application to moment convergence. Comput Math Appl. 50(5-6), 719-727 (2005). doi:10.1016/j.camwa.2005.04.015

31. Hao, Z-C: Note on the inequality of the arithmetic and geometric means. Pacific J Math. 143, 43-46 (1990)

32. Wang, C-L: Characteristics of nonlinear positive functionals and their applications. J Math Anal Appl. 95, 564-574 (1983). doi:10.1016/0022-247X(83)90126-9

33. Beckenbach, EF: A class of mean-value functions. Am Math Monthly. 57, 1-6 (1950). doi:10.2307/2305163

doi:10.1186/1029-242X-2011-77

Cite this article as: Tian: Extension of Hu Ke's inequality and its applications. Journal of Inequalities and Applications $20112011: 77$

\section{Submit your manuscript to a SpringerOpen ${ }^{\odot}$} journal and benefit from:

- Convenient online submission

Rigorous peer review

- Immediate publication on acceptance

- Open access: articles freely available online

- High visibility within the field

- Retaining the copyright to your article

Submit your next manuscript at $\gg$ springeropen.com 